\title{
Rheological behavior of a commercial AA5182 aluminum alloy during solidification
}

\author{
O. Ludwig ${ }^{\mathrm{a}, *}$, J.-M. Drezet ${ }^{\mathrm{a}, \mathrm{c}}$, P. Ménésès ${ }^{\mathrm{b}}$, C.L. Martin ${ }^{\mathrm{b}}$, M. Suéry ${ }^{\mathrm{b}}$ \\ ${ }^{a}$ Laboratoire de Simulation des Matériaux, Ecole Polytechnique Fédérale de Lausanne, Station 12, CH-1015 Lausanne, Switzerland \\ ${ }^{\mathrm{b}}$ Laboratoire Génie Physique et Mécanique des Matériaux, Institut National Polytechnique de Grenoble, UMR CNRS 5010, \\ ENSPG, BP46, 38402 Saint Martin d'Héres Cedex, France \\ ${ }^{\mathrm{c}}$ Calcom - ESI, Parc Scientifique, CH-1015 Lausanne, Switzerland
}

Received in revised form 11 July 2005

\begin{abstract}
During casting of aluminum alloys, the partially solidified material is submitted to thermally induced strains that can lead to severe casting defects, such as hot tearing. In this work, carried out in the frame of the European project VIR[CAST], the rheological behavior of a partially solidified AA5182 aluminum alloy has been investigated in order to provide constitutive equations to predict hot tearing in direct chill (DC) casting. Shear and tensile experiments have been performed using specific experimental devices and procedures previously designed for Al-Cu alloys. In the small strain $(<0.2)$ and high solid fraction $(>0.8)$ domain investigated here, the mushy zone is coherent. The stress-strain behavior is therefore dominated by the viscoplasticity of the solid phase, but exhibits a significant strain hardening. The behavior of the mushy zone is modeled by a compressible constitutive equation in which an internal variable, $C$, representing the state of cohesion of the mush, is introduced. The model accounts for solid fraction, stress state, strain rate and strain effects. The parameters that govern the evolution of $C$ with strain have been determined and appear to be comparable to those for $\mathrm{Al}-\mathrm{Cu}$ alloys.
\end{abstract}

(C) 2005 Elsevier B.V. All rights reserved.

Keywords: Rheological behavior; Constitutive equation; Aluminum alloys; Mushy zone; Solidification; Stress-strain modeling

\section{Introduction}

Aluminum alloys solidification processes, such as direct chill (DC) casting, laser welding or mould casting involve thermally induced deformations arising from the contraction that occurs during solidification and subsequent cooling. These strains can lead to severe casting defects, such as macrosegregation, porosity and hot tearing. In order to understand the formation of these defects, important modeling efforts have been undertaken recently directed towards the development of thermomechanical models for the solidifying alloy [1,2], rheological models of the mush [3-5] and hot tearing criteria [6]. In particular, constitutive equations of the mush have been developed taking into consideration the main aspects that are pertinent to the prediction of hot tears $[7,8]$. The mushy zone is treated as a compressible porous material saturated with liquid exhibiting some strain hardening. The effect of the liquid on the solid skeleton is taken into account via a hydrostatic pressure term. Moreover, the partial cohesion of

\footnotetext{
* Corresponding author. Tel.: +41 21693 3920; fax: +41 216935890.

E-mail address: olivier.ludwig@epfl.ch (O. Ludwig).
}

the mushy zone is introduced as an internal variable of the constitutive model. In order to develop relevant constitutive equations, experimental data are required during solidification for different stress states, solid fractions and strain rates. These data have been obtained recently in shear, compressive and tensile conditions for an Al-Cu alloy using devices specifically developed for this purpose [9-11].

In particular, the shear and tensile behaviors are believed to be of great importance for the generation of the casting defects, although compressive stress states can also play a role in specific regions of the casting. In addition, in DC casting the accumulated strain is relatively small (in any case less than 20\%) and the strain rates are low (less than $10^{-3} \mathrm{~s}^{-1}$ ). This paper describes briefly the theoretical aspects of the rheological model and presents the experimental identification and validation of the model parameters for an industrial AA5182 alloy in these two stress states.

\section{Rheological model of the mush}

The model that has been adopted for the rheological behavior of the mush is summarized hereafter. More details can be 
found in Refs. $[8,10]$. The mush is treated as a viscoplastic porous medium saturated with liquid. The effect of strain on the behavior of the partially solidified alloy is accounted for by introducing an internal variable $C$ that describes the state of cohesion of the mush. Since the evolution of this internal variable is considered as stress state dependent, it also accounts for the different mechanical response between tensile and compressive stress states. The constitutive equation is written on the effective solid stress tensor $\hat{\boldsymbol{\sigma}}_{\mathrm{s}}$ that allow us to take into account the mechanical effect of the liquid phase:

$\hat{\boldsymbol{\sigma}}_{\mathrm{s}}=\boldsymbol{\sigma}+p_{\mathrm{l}} \mathbf{I}$,

where $\boldsymbol{\sigma}$ is the total (applied) stress tensor and $p_{1}$ is the liquid interstitial pressure. An associated viscoplastic potential $\Omega$ is describing the relation between the effective solid stress tensor and the solid phase plastic strain rate tensor $\dot{\boldsymbol{\varepsilon}}_{\mathrm{s}}^{\mathrm{p}}$ (normality rule):

$\dot{\boldsymbol{\varepsilon}}_{\mathrm{s}}^{\mathrm{p}}=\frac{\partial \Omega}{\partial \hat{\boldsymbol{\sigma}}_{\mathrm{s}}}$

The external variables for the constitutive model are taken to be $\left(\hat{\boldsymbol{\sigma}}_{\mathrm{s}}, \dot{\boldsymbol{\varepsilon}}_{\mathrm{s}}^{\mathrm{p}}, T\right)$, where $T$ is the temperature. The internal variables are the triplet of scalars $\left(g_{\mathrm{s}}, C, s\right)$. The variable $g_{\mathrm{s}}$ is the solid volume fraction. The variable $C$ represents the cohesion of the solid skeleton and varies between zero and unity. The variable $s$ has the physical dimension of a stress and it represents an average isotropic resistance to plastic flow offered by the solid phase that constitutes the solid skeleton. In the present version of the model, $s$ is taken as constant $\left(s=s_{0}\right)$. This is because we are solely interested in the high temperature behavior of the solid, for which it is reasonable to assume that the plastic flow resistance is constant [7]. Here, the viscoplastic potential writes simply:

$\Omega=\Omega\left(\bar{P}_{\mathrm{s}}, \bar{\sigma}_{\mathrm{s}}, T, g_{\mathrm{s}}, C\right)$

where $\bar{P}_{\mathrm{S}}$ and $\bar{\sigma}_{\mathrm{s}}$ are the effective pressure on the solid skeleton and the Von Mises stress $\left(\bar{P}_{\mathrm{s}}=-\frac{1}{3} \operatorname{tr}\left(\hat{\boldsymbol{\sigma}}_{\mathrm{s}}\right)\right.$ and $\left.\bar{\sigma}_{\mathrm{s}}^{2}=\frac{3}{2} \operatorname{tr}\left(\mathbf{S}_{\mathrm{s}}: \mathbf{S}_{\mathrm{s}}\right)\right)$ with $\mathbf{S}_{\mathrm{s}}$ denoting the solid phase deviatoric effective stress tensor $\left(\boldsymbol{S}_{\mathrm{s}}=\hat{\boldsymbol{\sigma}}_{\mathrm{s}}+\bar{P}_{\mathrm{S}} \mathbf{I}\right)$. The expression of the viscoplastic potential proposed in [8] introduces both the softening effect of liquid saturated pores via a pressure dependent term and the effect of partial cohesion via the internal variable $C$ :

$\Omega=\frac{\dot{\varepsilon}_{0}}{(n+1)\left(C s_{0}\right)^{n}}\left(A_{2} \bar{P}_{\mathrm{s}}^{2}+A_{3} \bar{\sigma}_{\mathrm{s}}^{2}\right)^{\frac{n+1}{2}}$,

where $1 / n$ is the strain rate sensitivity (taken as temperature independent here), and $\dot{\varepsilon}_{0}=A \exp \left(-\frac{Q}{R T}\right)$ is the strain rate reference. The functions $A_{2}$ and $A_{3}$ depend solely on the solid fraction and are taken from the literature on dry porous viscoplastic materials $[12,13]$. Now applying Eq. (2) to the expression of the viscoplastic potential (Eq. (4)) leads to the expression of the strain rate tensor as implemented in the Finite Element code ABAQUS:

$\dot{\boldsymbol{\varepsilon}}_{\mathrm{s}}^{\mathrm{p}}=\frac{\dot{\varepsilon}_{0}}{\left(C s_{0}\right)^{n}}\left\{-\frac{A_{2}}{3} \bar{P}_{\mathrm{s}} \mathbf{1}+\frac{3}{2} A_{3} \mathbf{S}_{\mathrm{s}}\right\}\left\{A_{2} \bar{P}_{\mathrm{s}}^{2}+A_{3} \bar{\sigma}_{\mathrm{s}}^{2}\right\}^{\frac{n-1}{2}}$,

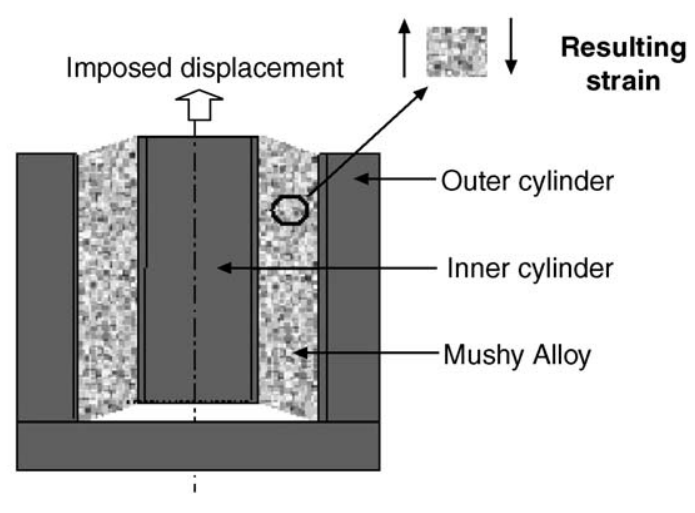

Fig. 1. Translation shear test.

For the fully solid material, for which $g_{\mathrm{s}}=1$ and $C=1$, the effective stress is the applied stress (Eq. (1)). Moreover, for $g_{\mathrm{s}}=1, A_{2}=0, A_{3}=1$, Eq. (5) reduces to the classical power-law of a purely viscoplastic dense material. Note also that when the liquid pressure effect is neglected, the effective stress reduces to the applied stress.

The evolution equation for the partial cohesion internal variable assumes that both the increase of $C$ by interlocking of dendrites and its decrease by rearrangement scale with a scalar measure of the macroscopic plastic strain rate $\dot{\varepsilon}_{\mathrm{e}}$ for any type of stress state so that:

$\frac{\mathrm{d} C}{\mathrm{~d} t}=\alpha\left(g_{\mathrm{s}}, X\right)\left(1-\frac{C}{C^{*}\left(g_{\mathrm{s}}, X\right)}\right) \dot{\varepsilon}_{\mathrm{e}}$,

where $\dot{\varepsilon}_{\mathrm{e}}=\sqrt{\frac{2}{3} \dot{\boldsymbol{\varepsilon}}_{\mathrm{s}}^{\mathrm{p}}: \dot{\boldsymbol{\varepsilon}}_{\mathrm{s}}^{\mathrm{p}}}$ and $\alpha\left(g_{\mathrm{s}}, X\right)$ and $C^{*}\left(g_{\mathrm{s}}, X\right)$ are two functions of the solid fraction and of the stress triaxiality $X=\bar{P}_{\mathrm{s}} / \bar{\sigma}_{\mathrm{s}}$. We have shown that in the case of pure shear $(X=0), \alpha\left(g_{\mathrm{s}}, 0\right)$ and $C^{*}\left(g_{\mathrm{s}}, 0\right)$ are increasing functions of the solid fraction and can be considered as rate independent in first approximation $[7,8]$.

\section{Experimental identification in pure shear}

The dependence of $\alpha$ and $C^{*}$ with the solid fraction is first determined in a pure shear stress state. The apparatus used for shearing the alloy in the solidification range is shown in Fig. 1. More details concerning the experimental procedure can be found in Ref. [9]. The alloy is initially melted in the container and the inner cylinder is inserted in the liquid that subsequently fills the gap between the two cylinders. The melt is then cooled down at a constant rate $\left(-5\right.$ to $\left.-20^{\circ} \mathrm{C} / \mathrm{min}\right)$ and the test is carried out at a given temperature. The solidification path is calculated with a numerical model accounting for back diffusion in the solid [14]. Shearing of the mush is imposed by the vertical translation of the inner cylinder at a constant speed and thereby constant strain rate. Grooves were machined on the surfaces of the two cylinders in order to avoid slippage.

The experimental determination of the characteristic functions $\alpha\left(g_{\mathrm{s}}, 0\right)$ and $C^{*}\left(g_{\mathrm{s}}, 0\right)$ is carried out on a grain refined AA5182, so that the dendrites can be considered as equiaxed. Therefore, isotropy of the behavior is assumed. The results of isothermal shear experiments are plotted in terms of Von 


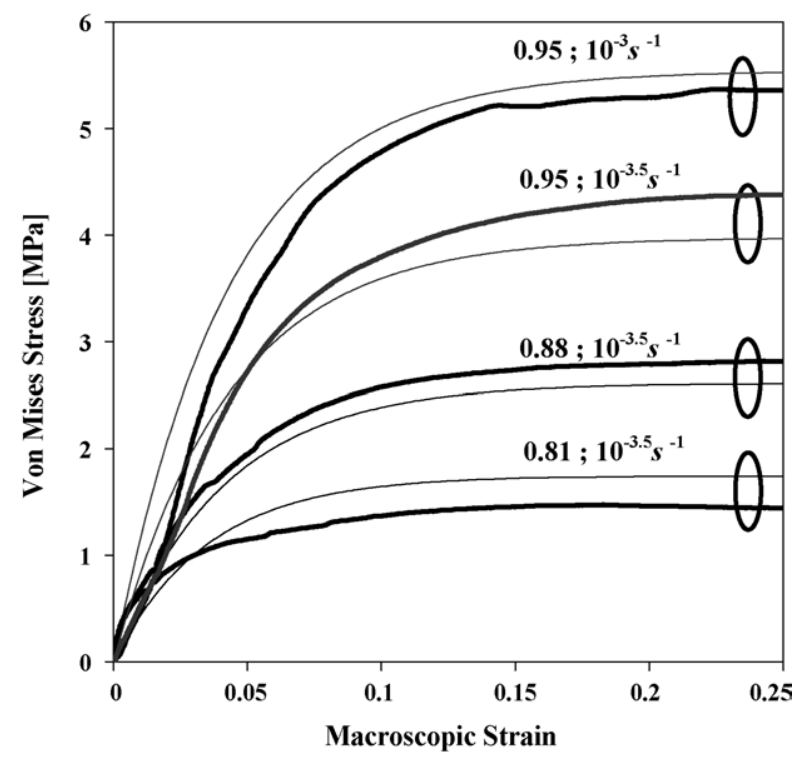

Fig. 2. Shear behavior of AA5182. Von Mises stress-strain curves for different solid fractions and strain rates. Experimental data (thick curves) and comparison with analytical predictions (thin curves).

Mises stress as a function of the macroscopic strain (Fig. 2). Stress-strain curves exhibit a gradual increase of stress with strain before reaching a viscoplastic plateau after 10-20\% strain. Since typical strains in DC casting do not exceed $10 \%$ in any case, modeling of the shear behavior requires to take into account this strain hardening. The rate effect on the maximum stress at a given temperature (solid fraction) as well as the effect of the solid fraction at a given strain rate are clearly demonstrated in Fig. 2. Stress increases both with increasing strain rate and increasing solid fraction. The strain rate sensitivity value $1 / n$ is characteristic of the fully solid phase behavior.

The determination of the functions $\alpha$ and $C^{*}$ that govern the shear behavior of the mushy zone at small strains is carried out by using isothermal experimental data. For isothermal and constant strain rate conditions, Eq. (6) can be integrated analytically. In order to describe the behavior of the solid phase (values of $s_{0}$, A, $Q$ and $n$ ), we use the results of Van Haaften et al. [15] given in Table 1.

A set of $\left(C^{*}, \alpha\right)$ values is determined for each couple of strain rate and solid fraction. Averaged values of $C^{*}$ and $\alpha$ are calculated for each solid fraction considering that these functions are strain rate independent. The functions:

$\alpha\left(g_{\mathrm{s}}, 0\right)=\alpha_{0}+\alpha_{1} \frac{g_{\mathrm{s}}{ }^{1 / 3}}{1-g_{\mathrm{s}}{ }^{1 / 3}}$ and $C^{*}\left(g_{\mathrm{s}}, 0\right)=1-\left(1-g_{\mathrm{s}}\right)^{\mathrm{p}}$

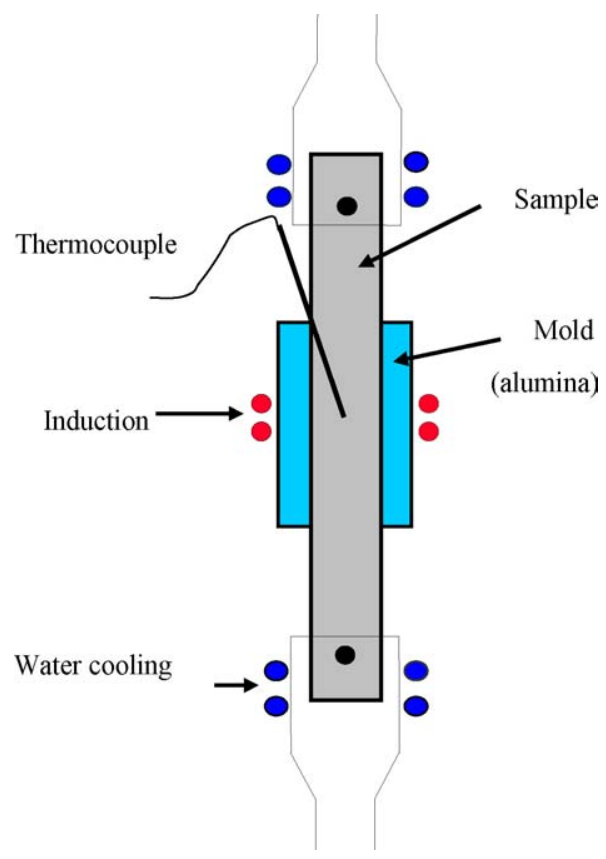

Fig. 3. Schematic of the tensile experimental set-up.

describe reasonably well the evolution of $C^{*}$ and $\alpha$ with the solid fraction in pure shear, with a small number of fitted parameters (values in Table 1). With these functions, good agreement between experimental stress-strain curves and predictions is obtained, as shown in Fig. 2.

\section{Tensile behavior}

The tensile behavior of AA5182 alloy during solidification is studied using the apparatus shown in Fig. 3.

The initially solid specimen is completely remelted by induction in its middle part, and then cooled at a controlled cooling rate of $1^{\circ} \mathrm{C} \mathrm{s}^{-1}$ until the temperature in the centre reaches a certain value in the solidification range. At this temperature, measured by a thermocouple, deformation is carried out at constant velocity $\left(0.02 \mathrm{~mm} \mathrm{~s}^{-1}\right)$. Fig. 4 shows typical stress-displacement curves at various solid fractions. Displacement is not transformed into strain since the length over which deformation takes place is not known. The curves show that maximum stress increases with increasing solid fraction. In addition, two different behaviors are observed: at solid fraction higher than 0.94 , the tensile stress is quite large before fracture but drops very rapidly at fracture; whereas for smaller solid fractions, it reaches lower values and decreases more gradually. This solid fraction of 0.94

Table 1

Rheological parameters of the model in the fully solid state $\left(s_{0}, A, Q, n\right)$ and in the mushy zone $\left(p, \alpha_{0}, \alpha_{1}, g_{\mathrm{s}}^{\text {coal }}, k\right)$ for Al-Cu and AA5182 alloys

\begin{tabular}{|c|c|c|c|c|c|c|c|c|c|}
\hline \multirow[t]{2}{*}{ Alloy } & \multicolumn{4}{|c|}{ Solid state parameters (Al-Cu: Ref. [10], AA5182: Ref. [15]) } & \multicolumn{5}{|c|}{ Mushy zone parameters(Al-Cu: Ref [10], AA5182: this work) } \\
\hline & $s_{0}(\mathrm{MPa})$ & $A\left(\mathrm{~s}^{-1}\right)$ & $Q(\mathrm{~kJ} / \mathrm{mol})$ & $n$ & $p$ & $\alpha_{0}$ & $\alpha_{1}$ & $g_{\mathrm{s}}^{\text {coal }}$ & $k$ \\
\hline AA5182 & 52 & $2.65 \times 10^{7}$ & 125 & 3.44 & 0.315 & 10.54 & 0.0632 & 0.94 & 100 \\
\hline
\end{tabular}

The solid state parameters are obtained from [15]. 


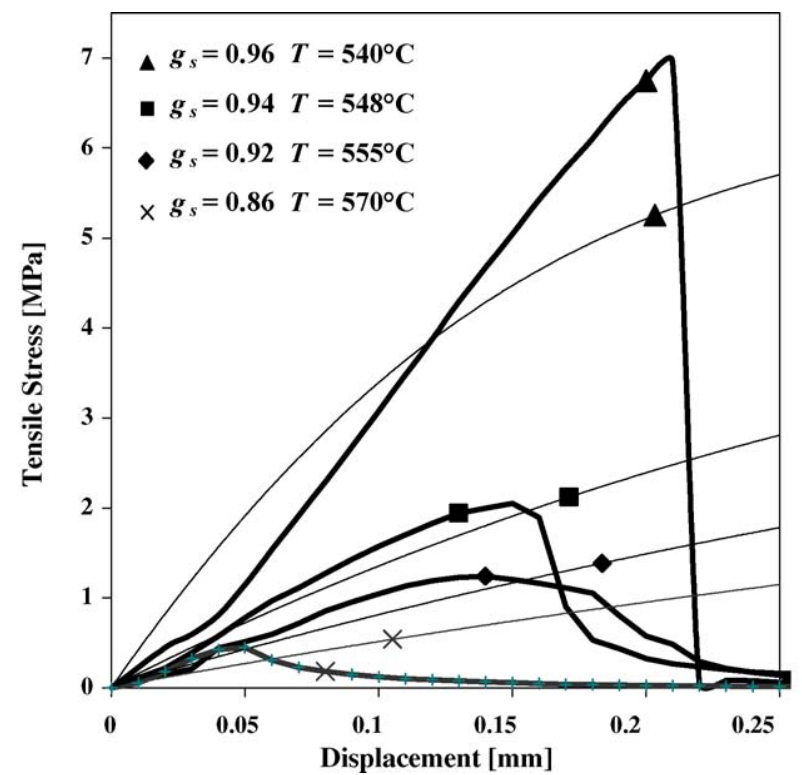

Fig. 4. Tensile behavior of AA5182 (stress vs. displacement) at various solid fractions. Experimental (thick curves) and numerical simulation results (thin curves).

seems therefore to correspond to the coalescence solid fraction at which solid bridges start to form extensively between the dendrites [16]. However, the material is brittle owing to the presence of residual liquid films.

Since there is a strong inhomogeneity of the temperature, solid fraction and, consequently, strains in the tensile sample, the model response is computed with the help of numerical simulations using the FEM code ABAQUS 6.4. The present model (Eqs. (5) and (6)) has been implemented using the user subroutine CREEP, [17], and neglecting the liquid pressure. The thermal field is considered as an input and does not evolve with time. As shown in Fig. 5, only one quarter of the specimen was modeled and axi-symmetric conditions were used. The axial displacement was imposed on the upper boundary of the computation domain and the sum of the reaction forces was recorded. The mesh was refined close to the center of the specimen where most of the deformation takes place owing to the solid fraction gradient. On the other hand, it was rather coarse in the fully solid region.

In order to account for coalescence in our model, the rheological function $\alpha$ is taken to be:

$\alpha\left(g_{\mathrm{s}}, X<0\right)=\alpha_{0}+\alpha_{1} \frac{g_{\mathrm{s}}^{1 / 3}}{1-g_{\mathrm{s}}^{1 / 3}} \exp \left(k\left(g_{\mathrm{s}}-g_{\mathrm{s}}^{\mathrm{coal}}\right)\right)$

where $g_{\mathrm{s}}^{\text {coal }}$ is the solid fraction at which coalescence starts. As demonstrated by Fig. 4, the experimental results are reasonably well reproduced for the following couple of parameters: $g_{\mathrm{s}}^{\text {coal }}=0.94$ and $k=100$. Note that the fracture of the sample is not (and cannot be) predicted, since the model does not include any fracture criterion. However, knowing the displacement at the onset of fracture from our experiments, the computation of the axial strain field for this displacement can

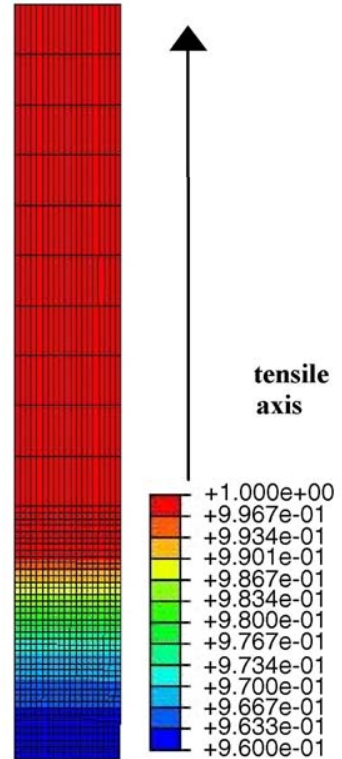

Solid fraction field

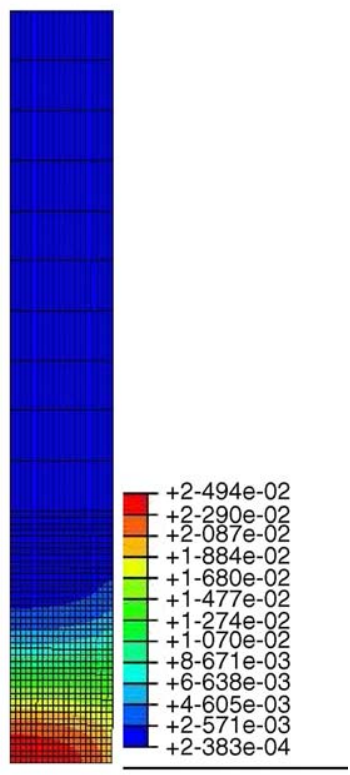

Axial strain field
Fig. 5. Solid fraction field (left) used for numerical simulations (input) and computed axial strain field (right) at the onset of fracture (result) for $g_{\mathrm{s}}=0.96$ in the center of the sample (one quarter of the sample section is shown).

help to determine the tensile ductility of the mushy zone (see Fig. 5).

\section{Comparison with Al-Cu alloys}

Qualitatively, the rheological behavior of AA5182 during solidification is very similar to that of $\mathrm{Al}-\mathrm{Cu}$ model alloys (see Refs. [9-11]), both in shear and tensile stress states. Quantitatively, the stress levels are comparable but stresses are, on the whole, higher for AA5182 than for Al-Cu in similar conditions. A first explanation for this difference is the fact that the fully solid AA5182 exhibits higher viscoplastic stresses at high temperature. However, this might not be a sufficient explanation: in the case of pure shear, the 5182 alloy exhibits also higher values of $\alpha$ and $C^{*}$, for the same solid fraction. In order to illustrate this case, Fig. 6 shows an example of comparison between the present experimental data and the model response for two different sets of parameters (see Table 1). Here,

1. Solid state parameters of AA5182, values of mushy zone parameters ( $\alpha$ and $C^{*}$ ) obtained for Al-Cu alloys (Ref. [10] and Table 1).

2. Solid state parameters of AA5182, mushy zone parameters obtained for AA5182 (Eq. (7), Table 1).

The agreement with the measured curves is good in the two sets of parameters, though much better with the second one (see Fig. 6). 


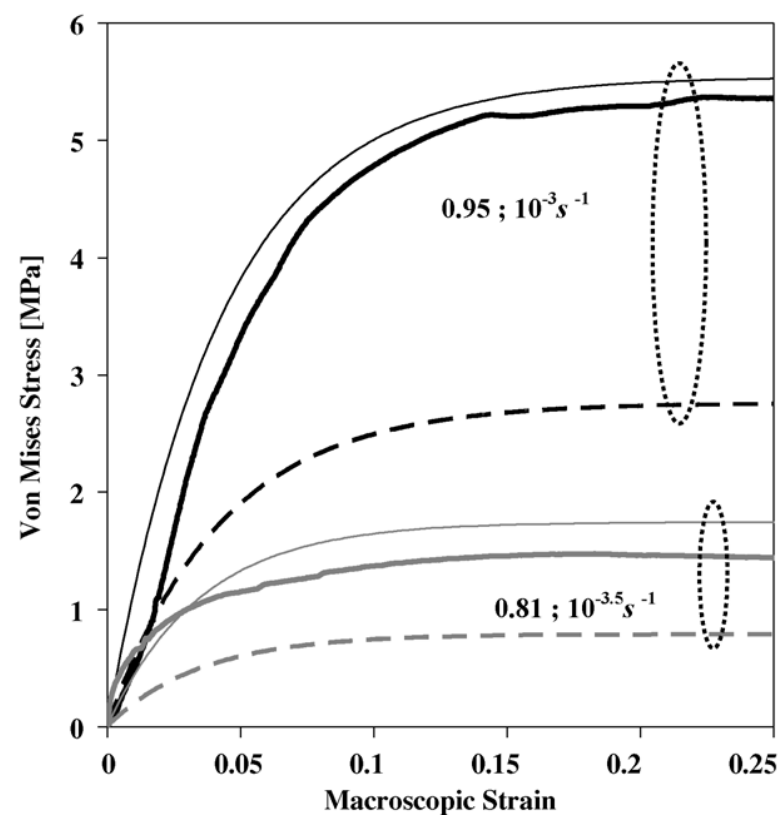

Fig. 6. Shear behavior of AA5182. Comparison between experimental and model results with two different sets of parameters and for two different solid fractions and strain rates. Thick solid lines: experimental curves; thin solid lines: model curves with AA5182 mushy zone parameters; dashed lines: model curves with $\mathrm{Al}-\mathrm{Cu}$ mushy zone parameters.

Concerning the tensile behavior, the values of the parameters $g_{\mathrm{s}}^{\text {coal }}$ and $k$, determined with the help of numerical simulations, are the same for the two alloys.

\section{Concluding remarks}

The rheological behavior of a partially solidified AA5182 has been investigated in shear and tension. Results are well reproduced by a phenomenological model previously developed for $\mathrm{Al}-\mathrm{Cu}$ alloys. This model introduces the concept of cohesion of the solid skeleton. The effect of cohesion on the rheological behavior is taken into account by the internal variable $C$. Its evolution is governed by two functions and accounts for strain effects and stress state dependence. Here, we have presented the model identification in two particular stress states, shear and tension. In order to generalize the model to any stress state, the dependence of $C$ with the triaxiality $X$ could be determined by making simple assumptions based on compressive testing (see Ref. [18]), in a similar way to what has been done for $\mathrm{Al}-\mathrm{Cu}$ alloys [10].

Moreover, the discussion in part 5 shows that, in a first approximation, the functions $\alpha$ and $C^{*}$ that govern the evolution of $C$ can be written with the same form and parameters whatever may be the alloy. Consequently, the rheological behavior of an alloy could be estimated using Al-Cu data, provided that the behavior of the solid alloy at high temperature is known (parameters $A, Q, n$ and $s_{0}$ ). However, if a good accuracy is needed, experimental identification of these functions is required for every specific alloy, at least in pure shear and tension. Moreover, the tensile ductility of the mushy zone might be very sensitive to the alloy composition and microstructure, which requires to perform tensile testing on every alloy if one is interested in the maximum tensile strain or strain rate that can sustain the mush. Such information would be needed to propose a hot tearing criterion $[6,19,20]$.

\section{Acknowledgements}

The authors would like to thank Benoit Commet, Alcan CRV, for providing the alloy and helpful discussions. They thank Guillaume Bard for his help in performing the shear experiments.

This research was carried out as part of the Fifth Framework Competitive and Sustainable Growth program, project GRD11999-10921 VIRCAST (Contract N G5RD-CT-2000-00153). It included the partners: Alcan Technology \& Management Ltd., Switzerland, Calcom SA, Switzerland, Elkem Aluminum ANS, Norway, École Polytechnique Fédérale de Lausanne, Switzerland, Corus, The Netherlands, Hydro Aluminum AS, Norway, Institut National Polytechnique de Grenoble, France, Institut National Polytechnique de Lorraine, France, Norwegian University of Science and Technology, Norway, Alcan France CRV, France, Hydro Aluminum Deutschland GmbH, Germany, and IFE, Norway and SINTEF, Norway, as major subcontractors. Funding by the European Community and by the Office Fédéral de l'Education et de la Science (Bern) for the Swiss partners is gratefully acknowledged.

\section{References}

[1] I. Farup, A. Mo, Metall. Mater. Trans. A 31A (2000) 1461-1472.

[2] M. M'hamdi, A. Mo, C.L. Martin, Metall. Mater. Trans. A 33A (2002) 2081-2093.

[3] C.L. Martin, D. Favier, M. Suéry, Int. J. Plasticity 13 (1997) 215-235.

[4] C.L. Martin, D. Favier, M. Suéry, Int. J. Plasticity 13 (1997) 237259

[5] C.L. Martin, D. Favier, M. Suéry, Int. J. Plasticity 15 (1999) 9811008 .

[6] M. Rappaz, J.-M. Drezet, M. Gremaud, Metall. Mater. Trans. A 30A (1998) 449-455.

[7] C.L. Martin, M. Braccini, M. Suéry, Mater. Sci. Eng. A325 (2002) 293-302.

[8] C.L. Martin, O. Ludwig, M. Suéry, in: H.A. Mang, F.G. Rammerstorfer, J. Eberhardsteiner (Eds.), World Congress on Computational Mechanics V, Vienna University of Technology, Austria, 2002.

[9] O. Ludwig, C.L. Martin, M. Suéry, in: Proceedings ICAA8, Mater. Sci. Forum. 396-402 (2002) 265-270.

[10] O. Ludwig, J.-M. Drezet, C.L. Martin, M. Suéry, Metall. Mater. Trans A 36A (2005) 1525-1535.

[11] O. Ludwig, B. Commet, J.-M. Drezet, C.L. Martin, M. Suéry, in: D.M. Stefanescu, J. Warren, M. Jolly, M. Krane (Eds.), Modeling of Casting, Welding and Advanced Solidification Processes, vol. X, The Minerals, Metals and Materials Society, Warrendale, USA, 2003, pp.183-190.

[12] A. Zavaliangos, L. Anand, J. Mech. Phys. Solids 41 (1993) 1087-1118.

[13] J.C. Michel, P. Suquet, J. Mech. Phys. Solids 40 (1992) 783-812.

[14] C. Sigli, L. Maenner, C. Sztur, R. Shahani, in: T. Sato, S. Kumai, T. Kobayashi, Y. Murakami (Eds.), Proceedings of International Conference on Aluminum Alloys, sixth ed., JILM, 1998, pp. 87-98.

[15] W.M. Van Haaften, B. Magnin, W.H. Kool, L. Katgerman, Metall. Mater. Trans. A 33A (2002) 1971-1980.

[16] M. Rappaz, J.-M. Drezet, P.-D. Grasso, A. Jacot, in: D.M. Stefanescu, J. Warren, M. Jolly, M. Krane (Eds.), Modeling of Casting, Welding and 
Advanced Solidification Processes, vol. X, The Minerals, Metals and Materials Society, Warrendale, USA, 2003, pp. 53-60.

[17] Abaqus Standard 6.4, Analysis User's Manual, HKS, RI, USA.

[18] J.-M. Drezet, O. Ludwig, M. M'Hamdi, H.-G. Fjaeer, C.L. Martin, Light metals, in: The Minerals Metals and Materials Society, Warrendale, USA, 2004, pp. 655-660.
[19] A. Mo, M. M'hamdi, H.G. Fjaer, in: D.M. Stefanescu, J. Warren, M. Jolly, M. Krane (Eds.), Modeling of Casting, Welding and Advanced Solidification Processes, vol. X, The Minerals, Metals and Materials Society, Warrendale, USA, 2003, pp.183-190.

[20] D.G. Suytno, V.I. Eskin, L. Savran, Katgerman, Metall. Mater. Trans. A 35A (2004) 3351-3361. 